\title{
A sufficient criterion for integrability of stochastic many-body dynamics and quantum spin chains
}

\author{
V. Popkov ${ }^{1,4}$, M.E. Fouladvand ${ }^{2,3}$ And G.M. Schütz ${ }^{1}$ \\ ${ }^{1}$ Institut für Festkörperforschung, Forschungszentrum Jülich, 52425 Jülich, Germany \\ ${ }^{2}$ Department of physics, Zanjan university, P.O. box 313, Zanjan, Iran \\ ${ }^{3}$ Institute for Studies in Theoretical Physics 8 Mathematics(IPM), P.O.Box 19395-5531, \\ Tehran,Iran \\ 4 Institute for Low Temperature Physics, 310164 Kharkov, Ukraine
}

\begin{abstract}
We propose a dynamical matrix product ansatz describing the stochastic dynamics of two species of particles with excluded-volume interaction and the quantum mechanics of the associated quantum spin chains respectively. The time-dependent algebra which is obtained from the action of the Markov generator of the exclusion process (or quantum Hamiltonian of the spin chain respectively) is given in terms of a set of quadratic relations. Analyzing the permutation consistency of the induced cubic relations we obtain sufficient conditions on the hopping rates (i.e., the quantum mechanical interaction constants) which allow us to identify integrable models. From the dynamical algebra we construct the quadratic algebra of Zamolodchikov type, associativity of which is a Yang Baxter equation. We also obtain directly from the dynamical matrix product ansatz the Bethe ansatz equations for the spectra of these models.
\end{abstract}

PACS numbers: 05.70.Ln, 75.10.Jm, 02.50.Ga 


\section{Introduction and summary of results}

The notion of integrability in classical stochastic interacting particle systems derives from the mapping to quantum spin systems being associated with a solution of the Yang-Baxter equation (YBE) [1, 34]. Classical particle occupation numbers are interpreted as quantum mechanical spin degrees of freedom. The generator of the infinitesimal Markovian time evolution thus becomes the quantum Hamiltonian of related quantum spin chain Đ. In its essence integrability refers to the existence of an infinite set of conservation laws which commute with the quantum Hamiltonian and which therefore govern the time evolution of the stochastic process. For integrable processes the Bethe ansatz and related methods complement probabilistic approaches [2] and allow for the derivation of critical exponents, density profiles and correlations, shock dynamics and other quantities of interest in the investigation of driven diffusive systems, reaction-diffusion processes and other systems both in and and far from equilibrium [3]. Thus integrability has emerged as a powerful tool in the study of Markov processes involving one or more species of particles which move and interact a one-dimensional lattice. Examples include the asymmetric exclusion process, spin-relaxation processes and reaction-diffusion systems.

A major difficulty in making use of integrability is posed by the problem of determining whether a given stochastic dynamics actually does correspond to an integrable system since the dynamics only determine the quantum Hamiltonian, but does not directly lead to the YangBaxter equation. The same problem occurs in the context of quantum spin chains alone, i.e., without reference to stochastic dynamics. Given the Hamiltonian densities as specified by the local interaction constants one would like to know whether the system is integrable or not. Even though in special cases an answer can be given by way of straightforward coordinate Bethe ansatz [6, 18], Baxterization [22] or by an integrability criterion due to Reshetikhin [7], a generic method for the construction of the Yang-Baxter equation directly from the quantum Hamiltonian is desirable. It is the purpose of this paper to provide such a method for stochastic interacting particle systems and their associated quantum spin chain Hamiltonians.

To this end we extend the dynamical matrix product ansatz (DMPA) [4, 5], originally developed for the single-species exclusion process and the associated spin- $1 / 2$ Heisenberg quantum chain, to two-species exclusion process with nearest-neighbor interaction and their associated spin 1 quantum chains. This is an approach where the action of the time evolution operator on an arbitrary state is rephrased in terms of a dynamical algebra of time-dependent operators with quadratic relations (see below). In this way we are able to answer two questions. The first is: Since the DMPA can be constructed for any stochastic process with nearest-neighbor interaction, what is special about the resulting algebra in integrable cases? We find that the special property is associativity which in its turn requires that cubic relations of the operators satisfy the YBE. The second question is: What choice of interaction parameters leads to an integrable case? Since our method is constructive and yields the YBE without a prior assumption on parameters, we obtain relations between the parameters which provide a sufficient criterion for integrability directly from the quantum Hamiltonian (i.e., without explicit reference to the infinite set of conservation laws and to an underlying transfer-matrix). In this way we recover not only a known family of integrable two-species exclusion processes which, using the Reshetikhin criterion, was believed to be the only one [8], but also a new family which had been studied earlier [31], but had not been known to be integrable.

This paper addresses rather different communities, viz. physicists and mathematicians

\footnotetext{
${ }^{1}$ The time evolution operators of nonequilibrium systems correspond to non-Hermitian variants of quantum spin systems, but in the cases we have in mind this does not affect the integrability.
} 
working on interacting particle systems on the one hand and on integrable models on the other hand. For the first group some of the remarks on quantum spin chains and integrable quantum field theory which appear in the paper may seem obscure and perplexing. Therefore we have tried to give a self-contained presentation of our results such that the paper can be followed even if all these allusions are being ignored. For a detailed and more pedagogical review we refer to [3]. Probabilists may also find the mathematically rigorous application of related quantum techniques in [11, 30] useful in this respect. We hope that for the second group of readers the non-standard (and non-rigorous) application of quantum integrability to classical stochastic dynamics pursued here will prove inspiring and not distracting. Complementary to the review [3] we refer to [2, 13, 12, 14 for the significance of stochastic interacting particle systems in a wider mathematical and physical perspective and to [15] specifically for driven lattice gases of the type studied here.

The paper is organized as follows. In Sec. II we first follow standard procedure [3] and introduce the quantum spin chain representation of the stochastic many-body dynamics. Then (Sec. III) we extend the DMPA developed in [4, 5] for the single-species exclusion process to the two-species case. The special case corresponding to symmetric hopping has been discussed recently in [29]. In Sec. IV we derive the YBE from the dynamical algebra and obtain the conditions on the parameters which guarantee that the YBE is satisfied. In Sec. V we derive the nested Bethe ansatz equations for the spectrum of the quantum Hamiltonian. The idea of proposing matrix states as special eigenstates of periodic quantum systems was first employed in [25, 24].

\section{Quantum Hamiltonian formalism}

The method of defining stochastic dynamics in terms of an imaginary-time Schrödinger equation with a "quantum Hamiltonian" as generator of the Markovian stochastic dynamics has been reviewed in detail in [3]. In order to be self-contained we present here a summary of what is required below.

Consider a configuration $\eta$ of particles on a lattice of $L$ sites. Here $\eta=\{\eta(1), \eta(2), \ldots, \eta(L)\}$ where $\eta(x)$ denotes the state of the system at site $x$ in terms of an occupation number for each permissible particle species. For definiteness we consider here only two-species exclusion processes where each lattice site is either occupied with a particle of species $A$ (local occupation number $\left.n_{x}^{A}=1\right)$ or species $B\left(n_{x}^{B}=1\right)$ or vacant respectively $\left(n_{x}^{A}=n_{x}^{B}=0\right)$. We may therefore define the "spin" variable $\eta(x)=n_{x}^{A}-n_{x}^{B} \in\{1,0,-1\}$ as a unique variable specifying site $x$. Physically one may interprete $\eta(x)$ e.g. as the charge of the particle occupying site $x$.

In the course of time random events take place which change a configuration $\eta$ of the system with rate $w_{\eta \rightarrow \eta^{\prime}}$. We define a stochastic process in terms of a master equation

$$
\frac{d}{d t} P(\eta ; t)=\sum_{\substack{\eta^{\prime} \in X \\ \eta^{\prime} \neq \eta}}\left[w_{\eta^{\prime} \rightarrow \eta} P\left(\eta^{\prime} ; t\right)-w_{\eta \rightarrow \eta^{\prime}} P(\eta ; t)\right]
$$

for the probability $P(\eta ; t)$ of finding the state $\eta$ at time $t$.

The idea of the "quantum Hamiltonian" formalism is to represent each of the possible particle configurations $\eta$ by a vector $|\eta\rangle$ which together with the transposed vectors $\langle\eta|$ form a basis of the vector space $X=\left(\mathbb{C}^{2}\right)^{\otimes L}$ and its dual respectively with scalar product $\left\langle\eta \mid \eta^{\prime}\right\rangle=$ $\delta_{\eta, \eta^{\prime}}$. We represent an empty site by the symbol 0 and occupied sites by $A, B$ and choose as 
local basis vectors

$$
|0\rangle=\left(\begin{array}{l}
1 \\
0 \\
0
\end{array}\right), \quad|A\rangle=\left(\begin{array}{l}
0 \\
1 \\
0
\end{array}\right), \quad|B\rangle=\left(\begin{array}{l}
0 \\
0 \\
1
\end{array}\right) .
$$

A state $\eta$ of the entire system is then represented by a tensor state $|\eta\rangle=\left|\eta_{1}\right\rangle \otimes \ldots \otimes \ldots\left|\eta_{L}\right\rangle$. Therefore the probability distribution is given by a state vector

$$
|P(t)\rangle=\sum_{\eta \in X} P(\eta ; t)|\eta\rangle .
$$

In this formalism one rewrites the master equation in the form of a Schrödinger equation in imaginary time,

$$
\frac{d}{d t} P(\eta ; t)=-\langle\eta|H| P(t)\rangle,
$$

where the off-diagonal matrix elements of $H$ are the (negative) transition rates $w_{\left(\eta^{\prime} \rightarrow(\eta\right.}$ between states and the diagonal entries are the inverse of the exponentially distributed life times of the states, i.e., the sum of all outgoig transition rates $w_{\eta \rightarrow \eta^{\prime}}$ from state $\eta$. In quantum mechanical interpretation $\eta(x)$ may be regarded as the $z$-component of the spin of an atom in a spin 1 state.

The master equation is linear in time and therefore has a formally simple solution. A state at time $t=t_{0}+\tau$ is given in terms of an initial state at time $t_{0}$ by

$$
\left|P\left(t_{0}+\tau\right)\right\rangle=\mathrm{e}^{-H \tau}\left|P\left(t_{0}\right)\right\rangle .
$$

The expectation value $\rho_{k}(t)=\left\langle s\left|n_{k}^{Z}\right| P(t)\right\rangle, Z=A, B$, for the $Z$-species density at site $x$ is given by the projection operator $n_{k}^{Z}$ which has value 1 if there is a particle of type $Z$ at site $k$ and 0 otherwise. The constant vector $\langle s|=\sum_{\eta \in X}\langle\eta|$ performs the average over all possible final states of the stochastic time evolution. The real part of the spectrum of $H$ is the set of all inverse relaxation times of the process. A non-vanishing imaginary part signals the presence of currents characterizing an non-equilibrium system. An equilibrium system satisfying detailed balance has a purely real relaxation spectrum and can always be mapped to a quantum system with real symmetric Hamiltonian $H$.

Here we consider diffusive systems with only hopping processes between neighboring sites. We assign hopping rates as follows

$$
\begin{aligned}
& A 0 \rightarrow 0 A \text { with rate } g_{A 0} \\
& 0 A \rightarrow A 0 \longrightarrow g_{0 A} \\
& B 0 \rightarrow 0 B \longrightarrow g_{B 0} \\
& 0 B \rightarrow B 0 \longrightarrow g_{0 B} \\
& A B \rightarrow B A \longrightarrow g_{A B} \\
& B A \rightarrow A B \longrightarrow g_{B A} .
\end{aligned}
$$


The transition matrix for the process defined on two lattice sites takes the form

$$
h=\left(\begin{array}{ccccccccc}
0 & 0 & 0 & 0 & 0 & 0 & 0 & 0 & 0 \\
0 & g_{0 A} & 0 & -g_{A 0} & 0 & 0 & 0 & 0 & 0 \\
0 & 0 & g_{0 B} & 0 & 0 & 0 & -g_{B 0} & 0 & 0 \\
0 & -g_{0 A} & 0 & g_{A 0} & 0 & 0 & 0 & 0 & 0 \\
0 & 0 & 0 & 0 & 0 & 0 & 0 & 0 & 0 \\
0 & 0 & 0 & 0 & 0 & g_{A B} & 0 & -g_{B A} & 0 \\
0 & 0 & -g_{0 B} & 0 & 0 & 0 & g_{B 0} & 0 & 0 \\
0 & 0 & 0 & 0 & 0 & -g_{A B} & 0 & g_{B A} & 0 \\
0 & 0 & 0 & 0 & 0 & 0 & 0 & 0 & 0
\end{array}\right) .
$$

In a periodic system with $L$ sites one therefore has

$$
H=\sum_{i=1}^{L} h_{i}
$$

where $h_{i}=\mathbf{1} \otimes \ldots \otimes h \otimes \ldots \otimes \mathbf{1}$ is the hopping matrix (7) acting on sites $i, i+1$ and $\mathbf{1}$ is the $3 \times 3$ unit matrix. For systems with open boundaries where particles are exchanged with external reservoirs the hopping matrix $h_{L}$ is replaced by suitably chosen boundary matrices $b_{1}, b_{L}$.

\section{Dynamical Matrix Product Ansatz}

In order to introduce the dynamical matrix product ansatz for a three-state system we define matrix valued vectors for a single site

$$
\begin{aligned}
& |\mathbf{A}\rangle=\left(\begin{array}{c}
E \\
D^{A} \\
D^{B}
\end{array}\right) \\
& |\mathbf{X}\rangle=\left(\begin{array}{c}
X^{0} \\
X^{A} \\
X^{B}
\end{array}\right)
\end{aligned}
$$

with time-dependent matrices $E, D^{A}, D^{B}$ and the time-dependent auxiliary matrices $X^{0, A, B}$. By taking a $L$-fold tensor product one obtains a matrix product state (MPS)

$$
|\mathbf{P}(t)\rangle=\left(\begin{array}{c}
E \\
D^{A} \\
D^{B}
\end{array}\right)^{\otimes L-1} \otimes\left(\begin{array}{c}
E Q \\
D^{A} Q \\
D^{B} Q
\end{array}\right)
$$

These matrices are chosen such that the MPS satisfies the master equation (4) for the hopping process defined by (6). Notice that there is the multiplication by some additional matrix $Q$ of the $L$-th term in (11). Choosing $Q$ to be time-independent, $\dot{Q}=0$, and following [1] this leads to an infinite-dimensional algebra of the matrices introduced above and their time derivatives which has quadratic relations given by

$$
\left(\frac{1}{2} \frac{d}{d t}+h\right)|\mathbf{A}\rangle \otimes|\mathbf{A}\rangle=|\mathbf{X}\rangle \otimes|\mathbf{A}\rangle-|\mathbf{A}\rangle \otimes|\mathbf{X}\rangle .
$$


Special care has to be given to the action of terms $h_{L}, h_{L-1}$ of the Hamiltonian (8) on $P(t)$, due to matrix $Q$ involved in (11). It can be shown, however, [20] that with $Q$ satisfying

$$
\left[Q, E^{-1} D^{Z}\right]=\left[Q, D^{Z} E^{-1}\right]=0 ; \quad Z=A, B .
$$

(we assume further invertibility of $E$ ), no extra relations arise in addition to (12). Note that in general we assume that $Q$ commutes neither with $D$ nor with $E$ separately, $[Q, E] \neq 0 ;[Q, D] \neq$ 0 .

Eqs. (12) contain nine relations, namely:

$$
\begin{aligned}
\frac{1}{2}(\dot{E} E+E \dot{E}) & =X^{0} E-E X^{0} \\
\frac{1}{2}\left(\dot{E} D^{A}+E \dot{D}^{A}\right)+g_{0 A} E D^{A}-g_{A 0} D^{A} E & =X^{0} D^{A}-E X^{A} \\
\frac{1}{2}\left(\dot{E} D^{B}+E \dot{D}^{B}\right)+g_{0 B} E D^{B}-g_{B 0} D^{B} E & =X^{0} D^{B}-E X^{B} \\
\frac{1}{2}\left(\dot{D}^{A} E+D^{A} \dot{E}\right)-g_{0 A} E D^{A}+g_{A 0} D^{A} E & =X^{A} E-D^{A} X^{0} \\
\frac{1}{2}\left(\dot{D}^{A} D^{A}+D^{A} \dot{D}^{A}\right) & =X^{A} D^{A}-D^{A} X^{A} \\
\frac{1}{2}\left(\dot{D}^{A} D^{B}+D^{A} \dot{D}^{B}\right)+g_{A B} D^{A} D^{B}-g_{B A} D^{B} D^{A} & =X^{A} D^{B}-D^{A} X^{B} \\
\frac{1}{2}\left(\dot{D}^{B} E+D^{B} \dot{E}\right)-g_{0 B} E D^{B}+g_{B 0} D^{B} E & =X^{B} E-D^{B} X^{0} \\
\frac{1}{2}\left(\dot{D}^{B} D^{A}+D^{B} \dot{D}^{A}\right)-g_{A B} D^{A} D^{B}-g_{B A} D^{B} D^{A} & =X^{B} D^{A}-D^{B} X^{A} \\
\frac{1}{2}\left(\dot{D}^{B} D^{B}+D^{B} \dot{D}^{B}\right) & =X^{B} D^{B}-D^{B} X^{B}
\end{aligned}
$$

If one finds matrices satisfying these relations one can calculate expectation values and probabilities $P(\eta, t)$ by taking a trace in the space on which the matrices act, i.e.

$$
|P(t)\rangle=\operatorname{Tr}|\mathbf{P}(t)\rangle / Z_{L}
$$

Here $Z_{L}=\operatorname{Tr} C^{L} Q$ with $C=E+D^{A}+D^{B}$. This is the sum of all unnormalized configurational probabilities and hence yields the correct normalization factor. For a given configuration $\eta$ the probability $P(\eta, t)$ is therefore obtained by taking the trace over a suitably chosen normalized product of $L$ matrices $D^{A}, D^{B}, E$. One represents an occupied (vacant) site by a time-dependent matrix $D^{Z}(E)$ in a string $D D D E D E E \ldots$ of $L$ such matrices. For illustration consider the probability $\left\langle n_{x}^{Z} n_{y}^{Z^{\prime}}(t)\right\rangle$ of finding two particles of species $Z, Z^{\prime}$ at sites $x, y$ in an otherwise empty system. One has

$$
\left\langle n_{x}^{Z}(t) n_{y}^{Z^{\prime}}(t)\right\rangle=\operatorname{Tr}\left(E^{x-1} D^{Z} E^{y-x-1} D^{Z^{\prime}} E^{L-y} Q\right) / Z_{L} .
$$

The initial state of the system is encoded in the initial values at $t=0$ of the matrices. This time-dependent algebra generalizes the stationary two-species case studied in detail in [10] and the time-dependent single-species algebra introduced in 四.

The algebra can be exploited either by studying explicit matrix representations or on a purely algebraic level [23]. Here we choose the second approach which has two objectives: (i) the elimination of the auxiliary operators in such a manner that information necessary 
to calculate the spectrum of $H$ as well as the configurational probabilities is retained, (ii) the elimination of the time-dependence from the algebraic relations. Our analysis consists of several steps, each of which leads to a reduction of the full algebra (14) - (22) to a smaller algebra with fewer generators and fewer relations.

Step 1:

Choosing

$$
\dot{E}=0
$$

one can without loss of generality satisfy (14) with the choice

$$
X^{0}=0
$$

One has the remaining eight relations

$$
\begin{aligned}
\frac{1}{2}\left(E \dot{D}^{A}\right)+g_{0 A} E D^{A}-g_{A 0} D^{A} E & =-E X^{A} \\
\frac{1}{2}\left(E \dot{D}^{B}\right)+g_{0 B} E D^{B}-g_{B 0} D^{B} E & =-E X^{B} \\
\frac{1}{2}\left(\dot{D}^{A} E\right)-g_{0 A} E D^{A}+g_{A 0} D^{A} E & =X^{A} E \\
\frac{1}{2}\left(\dot{D^{A}} D^{A}+D^{A} \dot{D}^{A}\right) & =X^{A} D^{A}-D^{A} X^{A} \\
\frac{1}{2}\left(\dot{D}^{A} D^{B}+D^{A} \dot{D}^{B}\right)+g_{A B} D^{A} D^{B}-g_{B A} D^{B} D^{A} & =X^{A} D^{B}-D^{A} X^{B} \\
\frac{1}{2}\left(\dot{D}^{B} E\right)-g_{0 B} E D^{B}+g_{B 0} D^{B} E & =X^{B} E \\
\frac{1}{2}\left(\dot{D}^{B} D^{A}+D^{B} \dot{D}^{A}\right)-g_{A B} D^{A} D^{B}-g_{B A} D^{B} D^{A} & =X^{B} D^{A}-D^{B} X^{A} \\
\frac{1}{2}\left(\dot{D}^{B} D^{B}+D^{B} \dot{D}^{B}\right) & =X^{B} D^{B}-D^{B} X^{B}
\end{aligned}
$$

A similar constraint was used in Ref. [28] for the study of the single-species case, leaving three instead of four relations. In 29] the choice $\dot{C}=0$ is made for the two-species case. In this case the sum of the auxiliary matrices is set to zero which has been shown to involve no loss of generality 33 .

Step 2:

Adapting the strategy of Ref. [4] we further assume that $E$ is invertible. This allows us to express the remaining auxiliary matrices $X_{A, B}$ in terms of the physical matrices $E, D^{A}, D^{B}$. Multiplying eqs. (27), (28),(29),(32) from the right and left resp. with $E^{-1}$ we find:

$$
\begin{aligned}
& 2 X^{A}=g_{A 0}\left(D^{A}+E^{-1} D^{A} E\right)-g_{0 A}\left(D^{A}+E D^{A} E^{-1}\right) \\
& 2 X^{B}=g_{B 0}\left(D^{B}+E^{-1} D^{B} E\right)-g_{0 B}\left(D^{B}+E D^{B} E^{-1}\right)
\end{aligned}
$$

Now there are six relations left. Two involve the time-derivatives of $D^{A}$ and $D^{B}$ respectively:

$$
\begin{aligned}
& \dot{D}^{A}=-g_{A 0}\left(D^{A}-E^{-1} D^{A} E\right)-g_{0 A}\left(D^{A}-E D^{A} E^{-1}\right) \\
& \dot{D}^{B}=-g_{B 0}\left(D^{B}-E^{-1} D^{B} E\right)-g_{0 B}\left(D^{B}-E D^{B} E^{-1}\right)
\end{aligned}
$$


Using also this yields four other equations:

$$
\begin{aligned}
g_{A 0} D^{A} E^{-1} D^{A} E+g_{0 A} E D^{A} E^{-1} D^{A} & =\left(g_{A 0}+g_{0 A}\right)\left(D^{A}\right)^{2} \\
g_{B 0} D^{B} E^{-1} D^{B} E+g_{0 B} E D^{B} E^{-1} D^{B} & =\left(g_{B 0}+g_{0 B}\right)\left(D^{B}\right)^{2} \\
g_{0 A} E D^{A} E^{-1} D^{B}+g_{B 0} D^{A} E^{-1} D^{B} E & =\left(g_{A 0}+g_{0 B}-g_{A B}\right) D^{A} D^{B}+g_{B A} D^{B} D^{A} \\
g_{0 B} E D^{B} E^{-1} D^{A}+g_{A 0} D^{B} E^{-1} D^{A} E & =\left(g_{B 0}+g_{0 A}-g_{B A}\right) D^{B} D^{A}+g_{A B} D^{A} D^{B} .
\end{aligned}
$$

Thus the originally quadratic problem of the time-evolution with nine relations for three physical operators and three auxiliary operators has been converted into the quartic problem (37) - (42) with six relations for the physical operators alone which have to be satisfied at all times. Notice that the relations (37), (38) are linear in the $D$-operators while the relations (39) - (42) are bilinear.

Step 3:

The linear relations (37), (38) are sufficient to describe the one-particle sector, i.e. the set of configurations with only one particle on the lattice. In order to eliminate the time-dependence we formally define "Fourier" components

$$
\begin{gathered}
\mathcal{D}_{p}^{Z}(t)=\sum_{k=-\infty}^{\infty} \mathrm{e}^{i p k} D_{k}^{Z}(t) \\
D_{k}^{A}(t)=\alpha^{k} E^{k-1} D^{A}(t) E^{-k} ; \quad D_{k}^{B}(t)=\beta^{k} E^{k-1} D^{A}(t) E^{-k}
\end{gathered}
$$

which have the property

$$
\begin{aligned}
& E^{-1} \mathcal{D}_{p}^{A}(t) E=\alpha \mathrm{e}^{i p} \mathcal{D}_{p}^{A}(t) \\
& E^{-1} \mathcal{D}_{p}^{B}(t) E=\beta \mathrm{e}^{i p} \mathcal{D}_{p}^{B}(t)
\end{aligned}
$$

Additionally we require that the matrix $Q$ from (11),(24) obeys

$$
\left[Q, D_{k}^{Z}(t)\right]=0
$$

which contains (13) as a special case and yields

$$
\left[Q, \mathcal{D}_{p}^{Z}(t)\right]=0
$$

Conversely one has

$$
D_{k}^{A, B}(t)=\frac{1}{2 \pi} \int_{-\pi}^{\pi} \mathrm{e}^{-i p k} \mathcal{D}_{p}^{A, B}(t) \mathrm{d} p
$$

The Fourier ansatz turns the time-dependent relations (37), (38) into two ordinary firstorder differential equations

$$
\dot{\mathcal{D}}_{p}^{A, B}(t)=-\epsilon_{p}^{A, B} \mathcal{D}_{p}^{A, B}(t)
$$

with the "dispersion relations"

$$
\begin{aligned}
& \epsilon_{p}^{A}=g_{0 A} \alpha^{-1} \mathrm{e}^{-i p}+g_{A 0} \alpha \mathrm{e}^{i p}-g_{0 A}-g_{A 0} \\
& \epsilon_{p}^{B}=g_{0 B} \beta^{-1} \mathrm{e}^{-i p}+g_{B 0} \beta \mathrm{e}^{i p}-g_{0 B}-g_{B 0} .
\end{aligned}
$$


In this way one can express the time-dependent matrix $\mathcal{D}_{p}^{A, B}(t)$ in terms of its initial value as

$$
\mathcal{D}_{p}^{A, B}(t)=\mathrm{e}^{-\epsilon_{p}^{A, B}} \mathcal{D}_{p}^{A, B}(0)
$$

In what follows we shall omit the time-argument in the initial matrices $D_{p}^{Z}(0)$.

In terms of the Fourier components (43) the four relations (39) - (42) turn into doubleintegral relations where the time-dependence is shuffled into an exponential. Because of (45) the quartic relations turn into quadratic relations. Using (49) with $p_{1}$ for the $A$ species and $p_{2}$ for the $B$ species in the third relation and vice versa in the fourth relation below one gets

$$
\begin{aligned}
0 & =\int \mathrm{d} p_{1} \int \mathrm{d} p_{2} a_{12} \mathcal{D}_{p_{1}}^{A} \mathcal{D}_{p_{2}}^{A} \mathrm{e}^{-\left(\epsilon_{p_{1}}^{A}+\epsilon_{p_{2}}^{A}\right) t} \\
0 & =\int \mathrm{d} p_{1} \int \mathrm{d} p_{2} b_{12} \mathcal{D}_{p_{1}}^{B} \mathcal{D}_{p_{2}}^{B} \mathrm{e}^{-\left(\epsilon_{p_{1}}^{B}+\epsilon_{p_{2}}^{B}\right) t} \\
0 & =\int \mathrm{d} p_{1} \int \mathrm{d} p_{2}\left[c_{12} \mathcal{D}_{p_{1}}^{A} \mathcal{D}_{p_{2}}^{B}-g_{B A} \alpha^{-1} \mathrm{e}^{-i p_{1}} \mathcal{D}_{p_{2}}^{B} \mathcal{D}_{p_{1}}^{A}\right] \mathrm{e}^{-\left(\epsilon_{p_{1}}^{A}+\epsilon_{p_{2}}^{B}\right) t} \\
0 & =\int \mathrm{d} p_{1} \int \mathrm{d} p_{2}\left[d_{12} \mathcal{D}_{p_{1}}^{B} \mathcal{D}_{p_{2}}^{A}-g_{A B} \beta^{-1} \mathrm{e}^{-i p_{1}} \mathcal{D}_{p_{2}}^{A} \mathcal{D}_{p_{1}}^{B}\right] \mathrm{e}^{-\left(\epsilon_{p_{1}}^{B}+\epsilon_{p_{2}}^{A}\right) t}
\end{aligned}
$$

with the functions

$$
\begin{aligned}
a_{12} \equiv a\left(p_{1}, p_{2}\right) & =g_{0 A} \alpha^{-2} \mathrm{e}^{-i p_{1}-i p_{2}}+g_{A 0}-\left(g_{0 A}+g_{A 0}\right) \alpha^{-1} \mathrm{e}^{-i p_{2}} \\
b_{12} & \equiv b\left(p_{1}, p_{2}\right)=g_{0 B} \beta^{-2} \mathrm{e}^{-i p_{1}-i p_{2}}+g_{B 0}-\left(g_{0 B}+g_{B 0}\right) \beta^{-1} \mathrm{e}^{-i p_{2}} \\
c_{12} & \equiv c\left(p_{1}, p_{2}\right)=g_{0 A} \alpha^{-1} \beta^{-1} \mathrm{e}^{-i p_{1}-i p_{2}}+g_{B 0}-\left(g_{0 B}+g_{A 0}-g_{A B}\right) \beta^{-1} \mathrm{e}^{-i p_{2}} \\
d_{12} \equiv d\left(p_{1}, p_{2}\right) & =g_{0 B} \alpha^{-1} \beta^{-1} \mathrm{e}^{-i p_{1}-i p_{2}}+g_{A 0}-\left(g_{0 A}+g_{B 0}-g_{B A}\right) \alpha^{-1} \mathrm{e}^{-i p_{2}}
\end{aligned}
$$

The four integral equations (54) - (57), obtained from (25) together with the assumption of existence of $E^{-1}$ and with the choice (26), form the basis of the subsequent analysis. As an intermediate summary we remark that at this point the only relations (out of originally nine) that remain are four bilinear relations (54) - (57). Correspondingly, all expectation values can be calculated from the initial matrices using (24), (49), (53). E.g. one has (see (24), (44), (49))

$$
\left\langle n_{x}^{Z}(t) n_{y}^{Z^{\prime}}(t)\right\rangle=\operatorname{Tr}\left(D_{x}^{Z} D_{y}^{Z^{\prime}} E^{L} Q\right) / Z_{L}=\iint d p_{1} d p_{2} \mathrm{e}^{-\left(\epsilon_{p_{1}}^{Z}+\epsilon_{p_{2}}^{Z^{\prime}}\right) t} \mathrm{e}^{-i p_{1} x-i p_{2} y} \operatorname{Tr}\left(\mathcal{D}_{p_{1}}^{Z} \mathcal{D}_{p_{2}}^{Z^{\prime}} E^{L} Q\right) / Z_{L}
$$

The only unknown quantities are time-independent matrix product elements of the form $\operatorname{Tr}\left(D_{p_{1}}^{Z}(0) D_{p_{2}}^{Z^{\prime}}(0) \ldots E\right.$ to be discussed below.

Step 4:

Before proceeding further a distinction between two different cases must be made. Since we assume that no particle species is completely immobile (i.e. the possibilities $g_{A 0}=g_{0 A}=0$ or $g_{B 0}=g_{0 B}=0$ are excluded) the functions $a_{12}$ and $b_{12}$ do not vanish identically. Indeed in the generic case, in the following referred to as case I, none of the four integrands (54) - (57) vanish identically. Only for the special case $0=g_{0 A}=g_{B 0}=g_{B A}=g_{A B}-g_{A 0}-g_{0 B}$ corresponding to $c_{12}=g_{B A}=0$ (or the equivalent case obtained by interchanging $A$ and $B$ species) the integrand in (56) (or (57) resp.) is zero. This is case II, to be treated separately.

\section{Case I:}


The relations (54) - (57) may be reformulated by splitting the integral into two parts as

$$
\int_{-\pi}^{\pi} \int_{-\pi}^{\pi} F_{p_{1}, p_{2}} \mathrm{~d} p_{1} \mathrm{~d} p_{2}=\int_{-\pi}^{\pi} \int_{-\pi}^{p_{1}} \ldots+\int_{-\pi}^{\pi} \int_{p_{1}}^{\pi} \ldots=I_{p 2<p 1}+I_{p 2>p 1}
$$

By changing the order of integration and interchanging $p_{1} \leftrightarrow p_{2}$ in the last term in (63) we obtain

$$
I_{p 2>p 1}=\int_{-\pi}^{\pi} \int_{-\pi}^{p_{2}} F_{p_{1}, p_{2}} \mathrm{~d} p_{2} \mathrm{~d} p_{1}=\int_{-\pi}^{\pi} \int_{-\pi}^{p_{1}} F_{p_{2}, p_{1}} \mathrm{~d} p_{1} \mathrm{~d} p_{2}
$$

Using (64.63) the relation (54) thus becomes

$$
\int_{-\pi}^{\pi} \mathrm{d} p_{1} \int_{-\pi}^{p_{1}} \mathrm{~d} p_{2}\left[a_{12} \mathcal{D}_{p_{1}}^{A} \mathcal{D}_{p_{2}}^{A}+a_{21} \mathcal{D}_{p_{2}}^{A} \mathcal{D}_{p_{1}}^{A}\right] \mathrm{e}^{-\left(\epsilon_{p_{1}}^{A}+\epsilon_{p_{2}}^{A}\right) t}=0
$$

In order to satisfy this for all times $t$ we require the integrand inside the brackets to vanish. This is a sufficient condition for satisfying also the original equations (54). A similar condition is obtained for (55). Relations (56), (57) can also be satisfied in this manner, but one has to require

$$
\epsilon_{p_{1}}^{A}+\epsilon_{p_{2}}^{B}=\epsilon_{p_{2}}^{A}+\epsilon_{p_{1}}^{B}
$$

This implies the constraints

$$
\begin{aligned}
\alpha g_{A 0} & =\beta g_{B 0} \\
\alpha^{-1} g_{0 A} & =\beta^{-1} g_{0 B}
\end{aligned}
$$

on the hopping rates.

In what follows we assume (67) and (68) to hold. One obtains the following four relations

$$
\begin{aligned}
a_{12} \mathcal{D}_{p_{1}}^{A} \mathcal{D}_{p_{2}}^{A} & =-a_{21} \mathcal{D}_{p_{2}}^{A} \mathcal{D}_{p_{1}}^{A} \\
b_{12} \mathcal{D}_{p_{1}}^{B} \mathcal{D}_{p_{2}}^{B} & =-b_{21} \mathcal{D}_{p_{2}}^{B} \mathcal{D}_{p_{1}}^{B} \\
c_{12} \mathcal{D}_{p_{1}}^{A} \mathcal{D}_{p_{2}}^{B}-g_{B A} \alpha^{-1} \mathrm{e}^{-i p_{1}} \mathcal{D}_{p_{2}}^{B} \mathcal{D}_{p_{1}}^{A} & =-c_{21} \mathcal{D}_{p_{2}}^{A} \mathcal{D}_{p_{1}}^{B}+g_{B A} \alpha^{-1} \mathrm{e}^{-i p_{2}} \mathcal{D}_{p_{1}}^{B} \mathcal{D}_{p_{2}}^{A} \\
d_{12} \mathcal{D}_{p_{1}}^{B} \mathcal{D}_{p_{2}}^{A}-g_{A B} \beta^{-1} \mathrm{e}^{-i p_{1}} \mathcal{D}_{p_{2}}^{A} \mathcal{D}_{p_{1}}^{B} & =-d_{21} \mathcal{D}_{p_{2}}^{B} \mathcal{D}_{p_{1}}^{A}+g_{A B} \beta^{-1} \mathrm{e}^{-i p_{2}} \mathcal{D}_{p_{1}}^{A} \mathcal{D}_{p_{2}}^{B}
\end{aligned}
$$

We stress that in these relations the time-dependence drops out. Therefore these are four static relations on the operators $\mathcal{D}_{p}^{Z}(0)$ which together with the two equations (53) and with (25), (26) form a sufficient (but not necessary) condition for satisfying the original algebra (12) on the manifold defined by (67),(68).

These algebraic relations can be written in a compact form by defining the operator valued two-component vector

$$
\mathcal{D}_{p}=\left(\begin{array}{c}
\mathcal{D}_{p}^{A} \\
\mathcal{D}_{p}^{B}
\end{array}\right)
$$

and the $4 \times 4$ matrix

$$
\Sigma\left(p_{1}, p_{2}\right)=\left(\begin{array}{cccc}
\sigma_{A A}^{A A} & 0 & 0 & 0 \\
0 & \sigma_{A B}^{A B} & \sigma_{A B}^{B A} & 0 \\
0 & \sigma_{B A}^{A B} & \sigma_{B A}^{B A} & 0 \\
0 & 0 & 0 & \sigma_{B B}^{B B}
\end{array}\right)
$$


with

$$
\begin{aligned}
\sigma_{A A}^{A A} & =-\frac{a_{21}}{a_{12}} \\
\sigma_{A B}^{A B} & =-\frac{\alpha \beta c_{21} d_{12}-g_{A B} g_{B A} \mathrm{e}^{-i p_{1}-i p_{2}}}{\alpha \beta c_{12} d_{12}-g_{A B} g_{B A} \mathrm{e}^{-2 i p_{2}}} \\
\sigma_{A B}^{B A} & =g_{B A} \beta \frac{\mathrm{e}^{-i p_{1}} d_{12}-\mathrm{e}^{-i p_{2}} d_{21}}{\alpha \beta c_{12} d_{12}-g_{A B} g_{B A} \mathrm{e}^{-2 i p_{2}}} \\
\sigma_{B A}^{A B} & =g_{A B} \alpha \frac{\mathrm{e}^{-i p_{1}} c_{12}-\mathrm{e}^{-i p_{2}} c_{21}}{\alpha \beta c_{12} d_{12}-g_{A B} g_{B A} \mathrm{e}^{-2 i p_{2}}} \\
\sigma_{B A}^{B A} & =-\frac{\alpha \beta d_{21} c_{12}-g_{A B} g_{B A} \mathrm{e}^{-i p_{1}-i p_{2}}}{\alpha \beta c_{12} d_{12}-g_{A B} g_{B A} \mathrm{e}^{-2 i p_{2}}} \\
\sigma_{A A}^{A A} & =-\frac{b_{21}}{b_{12}} .
\end{aligned}
$$

With these quantities relations $(\sqrt{69})-(\sqrt{72})$ read

$$
\mathcal{D}_{p_{1}} \otimes \mathcal{D}_{p_{2}}=\Sigma\left(p_{1}, p_{2}\right) \mathcal{D}_{p_{2}} \otimes \mathcal{D}_{p_{1}}
$$

We remark that the Fourier components $\mathcal{D}_{p}^{Z}(0)$ satisfy the algebra of creation operators in a 1+1-dimensional integrable quantum field theory [27]. The matrix $S=\Sigma P$ with the permutation operator $P$ acting on the two vector spaces may then be regarded as scattering matrix with matrix elements $S_{Y Y^{\prime}}^{Z Z^{\prime}}$ in row $Y Y^{\prime}$ and column $Z Z^{\prime}$,

$$
\mathcal{D}_{p_{1}}^{Z} \mathcal{D}_{p_{2}}^{Z^{\prime}}=S_{Y Y^{\prime}}^{Z Z^{\prime}} \mathcal{D}_{p_{2}}^{Y^{\prime}} \mathcal{D}_{p_{1}}^{Y}
$$

Applying (81) twice shows that $\Sigma$ to satisfy

$$
\Sigma\left(p_{1}, p_{2}\right) \Sigma\left(p_{2}, p_{1}\right)=1
$$

which is fulfilled without further constraints on the hopping rates. This is the analog of the field theoretical unitarity condition.

Case II:

Case II corresponds to the manifold

$$
g_{0 A}=g_{B 0}=g_{B A}=0, \quad g_{A B}=g_{A 0}+g_{0 B}
$$

which is the totally asymmetric two-species exclusion process

$$
\begin{array}{llll}
A 0 & \rightarrow 0 A & \text { with rate } & g_{A 0} \\
0 B & \rightarrow B 0 & & g_{0 B} \\
A B \rightarrow B A & & g_{A 0}+g_{0 B}
\end{array}
$$

investigated in [31]. By exchanging vacancies with $B$ particles this process is equivalent to the totally asymmetric process with both species hopping to the right, but

$$
\begin{array}{lll}
A 0 \rightarrow 0 A & \rightarrow \text { with rate } & g_{A 0}+g_{0 B} \\
B 0 \rightarrow 0 B & & g_{0 B} \\
A B \rightarrow B A & & g_{A 0} .
\end{array}
$$


On the manifold (84) relation (56) is satisfied identically. Without further constraints on the independent hopping rates $g_{A 0}, g_{0 B}$ and on the free parameters $\alpha, \beta$ one may satisfy (54), (55) by splitting the integrals into two domains as in case I, but by requiring the integrand in (57) to vanish without splitting of the integral. As in case I the resulting algebraic relations take the form (82) with

$$
\begin{aligned}
S_{A A}^{A A} & =-\frac{a_{21}}{a_{12}}=-\frac{\alpha-e^{-i p_{1}}}{\alpha-e^{-i p_{2}}} \\
S_{A B}^{B A}=S_{B A}^{A B} & =0 \\
S_{A B}^{A B} & =\beta \mathrm{e}^{i p_{2}} d_{21} / g_{A B}=\frac{\alpha \beta g_{A 0}+g_{0 B} e^{-i p_{1}-i p_{2}}}{\alpha g_{A B} e^{-i p_{2}}} \\
S_{B A}^{B A} & =\beta^{-1} \mathrm{e}^{-i p_{1}} g_{A B} / d_{12}=\frac{\alpha g_{A B} e^{-i p_{1}}}{\alpha \beta g_{A 0}+g_{0 B} e^{-i p_{1}-i p_{2}}} \\
S_{B B}^{B B} & =-\frac{b_{21}}{b_{12}}=-\frac{e^{-i p_{1}}\left(\beta-e^{-i p_{2}}\right)}{e^{-i p_{2}}\left(\beta-e^{-i p_{1}}\right)} .
\end{aligned}
$$

Notice that the corresponding $S$-matrix is diagonal. The unitarity condition (83) holds. The crossing-symmetry relation is generally not satisfied.

\section{Dynamical algebra and Yang-Baxter equations}

The quadratic relations discussed above are sufficient to describe only the sectors with one or two particles respectively. In order to study the $n$-body problem within this approach one has to make sure that the expectation values expressed in terms of the quantities $\operatorname{Tr}\left(D_{p_{1}}^{Z} D_{p_{2}}^{Z^{\prime}} D_{p_{3}}^{Z^{\prime \prime}} \ldots E^{L}\right) / Z_{L}$ automatically satisfy the original master equation, irrespective of the contours of integration over the pseudo momenta $p_{i}$. This can only be ensured by requiring associativity of the algebra defined by (81) which in turn implies conditions on the properties of the $\Sigma$-matrix (74) or the $S$-matrix respectively.

The matrix $\Sigma$ acts like a generalized permutation operator on the tensor product of vector spaces defined by (73). Hence associativity implies that different orders of permutations must lead to the same final result. This in not trivial since the vector components are noncommutative objects. Let us define $\Sigma^{(1)}\left(p_{1}, p_{2}\right)=\Sigma\left(p_{1}, p_{2}\right) \otimes \mathbf{1}$ with the $2 \times 2$ identity matrix 1 acting trivially on the third subspace of a tensor vector $\mathcal{D}_{p_{1}} \otimes \mathcal{D}_{p_{2}} \otimes \mathcal{D}_{p_{3}}$. Analogously we define $\Sigma^{(2)}$ as acting trivially on the first subspace. Applying (81) in the order $1 \leftrightarrow 2,2 \leftrightarrow 3$, $1 \leftrightarrow 2$ yields

$$
\mathcal{D}_{p_{1}} \otimes \mathcal{D}_{p_{2}} \otimes \mathcal{D}_{p_{3}}=\Sigma^{(1)}\left(p_{1}, p_{2}\right) \Sigma^{(2)}\left(p_{1}, p_{3}\right) \Sigma^{(1)}\left(p_{2}, p_{3}\right) \mathcal{D}_{p_{3}} \otimes \mathcal{D}_{p_{2}} \otimes \mathcal{D}_{p_{1}}
$$

On the other hand, choosing the order of permutations as $2 \leftrightarrow 3,1 \leftrightarrow 2,2 \leftrightarrow 3$ one arrives at

$$
\mathcal{D}_{p_{1}} \otimes \mathcal{D}_{p_{2}} \otimes \mathcal{D}_{p_{3}}=\Sigma^{(2)}\left(p_{2}, p_{3}\right) \Sigma^{(1)}\left(p_{1}, p_{3}\right) \Sigma^{(2)}\left(p_{1}, p_{2}\right) \mathcal{D}_{p_{3}} \otimes \mathcal{D}_{p_{2}} \otimes \mathcal{D}_{p_{1}}
$$

Associativity therefore implies

$$
\Sigma^{(1)}\left(p_{1}, p_{2}\right) \Sigma^{(2)}\left(p_{1}, p_{3}\right) \Sigma^{(1)}\left(p_{2}, p_{3}\right)=\Sigma^{(2)}\left(p_{2}, p_{3}\right) \Sigma^{(1)}\left(p_{1}, p_{3}\right) \Sigma^{(2)}\left(p_{1}, p_{2}\right) .
$$

This is set of 64 equations for the hopping rates which must be satisfied for all $p_{i}$. (Extra solutions for special values are discussed below). In terms of the elements of the $S$-matrix the 
relations (94) read

$$
S_{i j}^{i^{\prime \prime} j^{\prime \prime}}\left(p_{1}, p_{2}\right) S_{i^{\prime \prime} k}^{i^{\prime} k^{\prime \prime}}\left(p_{1}, p_{3}\right) S_{j^{\prime \prime} k^{\prime \prime}}^{j^{\prime} k^{\prime}}\left(p_{2}, p_{3}\right)=S_{j k}^{j^{\prime \prime} k^{\prime \prime}}\left(p_{2}, p_{3}\right) S_{i k^{\prime \prime}}^{i^{\prime \prime} k^{\prime}}\left(p_{1}, p_{3}\right) S_{i^{\prime \prime} j^{\prime \prime}}^{i^{\prime} j^{\prime}}\left(p_{1}, p_{2}\right)
$$

These are the Yang-Baxter equations with the usual Einstein convention of summing over internal indices. In another compact form they may be written

$$
S_{12} S_{13} S_{23}=S_{23} S_{13} S_{12}
$$

where $S_{i j}$ is the $S$-matrix acting on spaces $i, j$ as a function of the pseudomomenta $p_{i}, p_{j}$. If these equations are satisfied no extra constraints arise from consistency relations involving more than three operators $\mathcal{D}$.

Since in case II the $S$-matrix is diagonal the YBE is satisfied automatically, i.e., there is no further constraint on the hopping rates which would be required for integrability. In case I some more discussion is necessary. The various conditions on the rates arising from the YBE can be obtained analytically using the software packages mathematica or maple. Due to the fact that there is a charge conservation $\left(S_{i j}^{i^{\prime} j^{\prime}}=0\right.$, unless $\left.i+j=i^{\prime}+j^{\prime}\right)$, only the equations (95) with $i+j+k=i^{\prime}+j^{\prime}+k^{\prime}$ are nonzero, which leaves 20 equations. Out of them 6 equations are satisfied trivially. The remaining 14 equations are pairwise equivalent, leaving only 7 independent relations.

It is convenient to pick one of the those independent equations, derive the arising constraints on the rates (if any), use this constraint in the remaining equations and then to iterate until all equations are satisfied. The solutions that we have found can be classified according to the values of the hopping rates $g_{Z Z^{\prime}} ; Z, Z^{\prime}=0, A, B$. The crossing-symmetry relation (written as $S_{\alpha \beta}^{\alpha^{\prime} \beta^{\prime}}(p, q)=S_{\beta \alpha^{\prime}}^{\beta^{\prime} \bar{\alpha}}(-p,-q), \bar{A}=B, \bar{B}=A$ for the spectral-parameter dependent $S$ matrix (102) ) is in general not satisfied. In that respect the algebra (82) is not exactly the Zamolodchikov algebra [27], but the one with some field-theoretical restrictions relaxed.

\subsection{All hopping rates nonzero}

This group consist of a solution with

$$
\text { (a) } \alpha=\beta ; \quad g_{A 0}=g_{B 0}=g_{A B}=g ; \quad g_{0 A}=g_{0 B}=g_{B A}=h
$$

and those obtained by relabeling the particles/holes, $A \leftrightarrow B, B \leftrightarrow 0$ :

$$
\begin{gathered}
\text { (b) } \alpha=\beta ; \quad g_{A 0}=g_{B 0}=g_{B A}=g ; \quad g_{0 A}=g_{0 B}=g_{A B}=h \\
\text { (c) } \alpha=\beta h / g ; \quad g_{A 0}=g_{0 B}=g_{A B}=g ; \quad g_{0 A}=g_{B 0}=g_{B A}=h
\end{gathered}
$$

Note that other reshuffling of labels will not result in new sets of rates, e.g. relabeling $A \leftrightarrow 0$ in (97) gives again (97). We have checked that all the cases (97-99) lead to the $S$-matrix of the same type given below. Therefore only the solution (97) will be considered in detail.

Note that constants $\alpha, \beta$ are defined up to common factor, since one can redefine $\alpha \mathrm{e}^{i p} \rightarrow \mathrm{e}^{i p}$. Therefore in case (97) one can consider $\alpha=\beta=1$ without losing generality.

The corresponding $S$-matrix

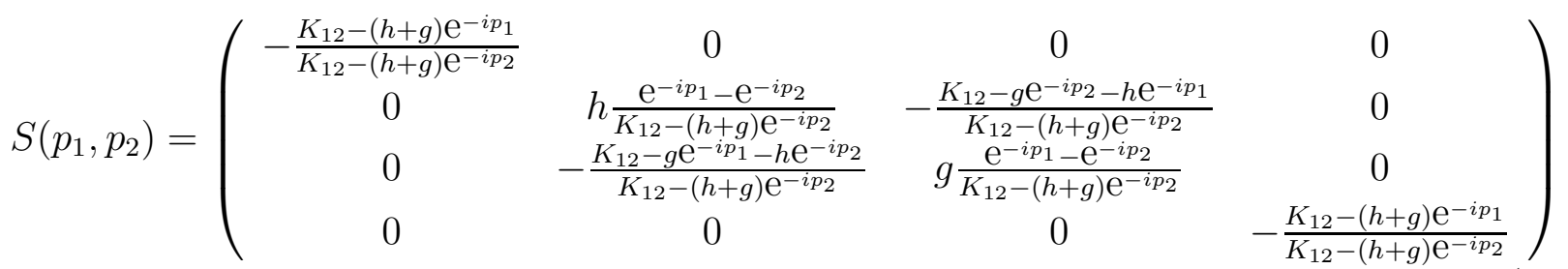




$$
K_{12}=h \mathrm{e}^{-i p_{1}-i p_{2}}+g
$$

after the transformation

$$
\mathrm{e}^{-i p}=\mathrm{e}^{\eta} \frac{\sinh (\lambda)}{\sinh (\lambda+\eta)} ; \quad \mathrm{e}^{\eta}=\sqrt{\frac{g}{h}}
$$

is parametrized to a difference form $S\left(p_{1}, p_{2}\right)=S\left(\lambda_{1}-\lambda_{2}\right)$. The precise form of $S$ as a function of a spectral parameter $\lambda$ [26] $S(\lambda)$ is noteworthy (sh $\equiv \sinh$ ):

$$
\operatorname{sh}(\lambda+\eta) \quad S(\lambda)=\left(\begin{array}{cccc}
\operatorname{sh}(\lambda-\eta) & 0 & 0 & 0 \\
0 & \mathrm{e}^{-\eta} \operatorname{sh}(\lambda) & -\mathrm{e}^{\lambda} \operatorname{sh}(\eta) & 0 \\
0 & -\mathrm{e}^{-\lambda} \operatorname{sh}(\eta) & \mathrm{e}^{\eta} \operatorname{sh}(\lambda) & 0 \\
0 & 0 & 0 & \operatorname{sh}(\lambda-\eta)
\end{array}\right)
$$

The anisotropy parameter plays a special role in the theory of 6-vertex model [26]

$$
\Delta=\frac{S_{11}^{11} S_{22}^{22}+S_{21}^{21} S_{12}^{12}-S_{21}^{12} S_{12}^{21}}{2 \sqrt{S_{11}^{11} S_{22}^{22} S_{21}^{21} S_{12}^{12}}}=\cosh (\eta)
$$

If $h=g$, the transformation (101) is to be substituted with $\mathrm{e}^{-i p}=(\lambda+i / 2) /(\lambda-i / 2)$, to retrieve the well-known rational solution of $\mathrm{YBE} S(\lambda) \approx \lambda I+i P, P$ being a permutation operator.

Note however that for the general case $h \neq g$, the solution (102) differs from the usual trigonometric one due to the spectral parameter dependence in the adiagonal elements. This dependence can be removed however by a similarity transformation, and in addition it plays

no role in the equations for the spectrum. Note also that the sum of S-matrix elements along each column is the same for each column, so that $S$-matrix is stochastic.

Finally, note that the choice of rates (97) was listed as an integrable case in [9].

\subsection{Some hopping rates zero}

Note that Eq.(45,46) imply that both auxiliary constants $\alpha, \beta$ are nonzero. In what follows, we require this to hold, $\alpha \beta \neq 0$.

1. $g_{A B}=g_{B A}=0, g_{A 0}=g_{B 0}, g_{0 A}=g_{0 B}, \alpha=\beta$.

Again as in the case (97), one can choose $\alpha=1$. The corresponding $S$-matrix is proportional to a permutation operator, $S_{12}=f_{12} P$, defined as $P_{i^{\prime} j^{\prime}}^{i j}=\delta_{i j^{\prime}} \delta_{i^{\prime} j}$. This is a tracer diffusion process. One can imagine it as usual exclusion process with particles $A$ and $B$ having identical dynamics, but different colors [32]. The proportionality coefficient is

$$
f_{12}=\left(f_{21}\right)^{-1}=-\frac{g_{A 0}-\left(g_{0 B}+g_{A 0}\right) \mathrm{e}^{-i p_{1}}+g_{0 B} \mathrm{e}^{-i p_{1}-i p_{2}}}{g_{A 0}-\left(g_{0 B}+g_{A 0}\right) \mathrm{e}^{-i p_{2}}+g_{0 B} \mathrm{e}^{-i p_{1}-i p_{2}}}
$$

2. $g_{B 0}=g_{A 0}=g_{B A}=0, g_{0 A}=g_{0 B} \alpha / \beta, g_{A B}=g_{0 B}(\beta-\alpha) / \beta$.

Note that $g_{0 B}=g_{0 A}+g_{A B}$, and since the constants $\alpha, \beta$ are arbitrary, after relabeling holes and particles $A \leftrightarrow 0$ we obtain the set of constants considered already in Case II. However since it is the different physical system, we shall list it independently here.

The $S$-matrix is: 


$$
-S\left(p_{1}, p_{2}\right)=\left(\begin{array}{cccc}
\frac{1-\alpha \mathrm{e}^{i p_{2}}}{1-\alpha \mathrm{e}^{i p_{1}}} & 0 & 0 & 0 \\
0 & 0 & \frac{1-\alpha \mathrm{e}^{i p_{2}}}{1-\alpha \mathrm{e}^{i p_{1}}} & 0 \\
0 & \frac{1-\alpha \mathrm{e}^{i p_{2}}}{1-\alpha \mathrm{e}^{i p_{1}}} & \frac{\alpha(\alpha-\beta)}{\beta} \frac{\mathrm{e}^{i p_{2}}-\mathrm{e}^{i p_{1}}}{\left(1-\alpha \mathrm{e}^{i p_{1}}\right)^{2}} & 0 \\
0 & 0 & 0 & \frac{1-\beta \mathrm{e}^{i p_{2}}}{1-\beta \mathrm{e}^{i p_{1}}}
\end{array}\right)
$$

The $S$-matrix above can be viewed as the 5 -vertex model. The characteristic parameter $\Delta$ for this case is 21]:

$$
\Delta=\frac{S_{A A}^{A A} S_{B B}^{B B}-S_{A B}^{B A} S_{B A}^{A B}}{S_{B B}^{B B} S_{B A}^{B A}}=\frac{b\left(a-\mathrm{e}^{-i p_{2}}\right)}{a\left(b-\mathrm{e}^{-i p_{2}}\right)}
$$

depends on the value of $p_{2}$. In the spirit of (113) the solution of the problem can be reduced to one of finding the eigen-values of the transfer-matrix with site-dependent weights. The essential property of the above $S\left(p_{1}, p_{2}\right)$-matrix is that it cannot be transformed into a form containing only the difference of the spectral parameters, unlike the case (102). The corresponding Bethe equations are given in the next section. Alternatively, one can proceed by relabeling particles and holes $A \leftrightarrow 0$ and then using (111). We assume that $\alpha \neq \beta$ in (105) since $\alpha=\beta$ falls into special tracer diffusion case (solution 1 ).

3. $g_{0 A}=g_{0 B}=g_{B A}=0, g_{B 0}=g_{A 0} \alpha / \beta, g_{A B}=g_{A 0}(\beta-\alpha) / \beta$. This model can be transformed into the previous one by renaming $A \leftrightarrow B$ and reversing the direction of particle motion $g_{0 A} \leftrightarrow g_{A 0}$, etc.. The $S$-matrix is of type (105). Consequently the equations for the spectrum are analogous to (118) and we shall not separately list them here.

The cases listed above exhaust the list of the nontrivial solutions of the YBE for two species of particles.

\section{$5 \quad$ Spectral equations}

Because of particle number conservation eigenstates and their eigenvalues can be classified according to number $N^{Z}$ of particles of each species that move on the ring. We denote by $N=N^{A}+N^{B}$ the total number of particles. The quantity $Q=N^{A}-N^{B}$ shall be referred to as charge.

$N=0$ :

This is the empty lattice. Since this is obviously an invariant state under the stochastic dynamics. Hence the single eigenvalue

$$
\epsilon=0
$$

of $H$ in this sector vanishes by construction.

$N=1:$

In order to obtain the relaxation spectrum from the eigenvalues

$$
\epsilon_{p}^{Z}=g_{0 Z} \gamma^{-1} \mathrm{e}^{-i p}+g_{Z 0} \gamma \mathrm{e}^{i p}-g_{0 Z}-g_{Z 0}
$$

where $\gamma=\alpha, \beta, Z=A, B$ depending on the species of a single-particle system on a finite lattice, one uses the Fourier ansatz and the solution (53). This requires calculating $\mathcal{F}_{Z}(p)=$ 
$\operatorname{Tr}\left(\mathcal{D}_{Z}(p) E^{L} Q\right)$ at time $t=0$. Because of the cyclic property of the trace the equation $\mathcal{F}_{Z}(p)=$ $\gamma^{L} \mathrm{e}^{i p L} \mathcal{F}_{Z}(p)$ must be satisfied. This yields the spectral equation

$$
\gamma^{L} \mathrm{e}^{i p L}=1
$$

and thus fixes the allowed values of the pseudomomenta $p=2 \pi n / L+i \ln \gamma$. We stress that this quantization of the pseudomomentum is not a property of the matrices $\mathcal{D}_{Q}(p)$ themselves. It appears only as a result of taking the trace in the one-particle sector. For expectation values in higher sectors we shall obtain different quantization constraints. We remark that for calculating only the spectrum the normalization factor $Z_{L}$ is not required.

$N \geq 2:$

In the case when there are $n$ particles of both types $A$ and $B$ in the system, the averages are written in terms of quantities

$$
\mathcal{F}^{Z_{1}, \ldots Z_{N}}\left(p_{1}, \ldots p_{N}\right)=\operatorname{Tr}\left(\mathcal{D}^{Z_{1}}\left(p_{1}\right) \ldots \mathcal{D}^{Z_{N}}\left(p_{N}\right) E^{L} Q\right)
$$

Now we have to distinquish between different integrable models.

\section{Case II}

The easiest to handle is the Case II, where $S$-matrix is diagonal.

Commuting the $\mathcal{D}\left(p_{k}\right)$ through around the "circle" inside the trace in (110), and using the algebra (82), one gets, using the trace property:

$$
\prod_{j \neq k}^{N} S_{Z_{k} Z_{j}}^{Z_{k} Z_{j}}\left(p_{k}, p_{j}\right) \mathrm{e}^{i p_{k} L}=1, \quad k=1, \ldots N
$$

with $S$-matrix elements written in (87-91). $\alpha, \beta$ can be set both to 1 by simple rescaling of quasiimpulses of $A$ and $B$ particles. It can be shown [17] that applying the coordinate Bethe Ansatz directly to the process (85) yields the same result (111).

\section{Case I, all nonzero hopping rates}

Commuting the $\mathcal{D}\left(p_{1}\right)$ through around the "circle" inside the trace in (110), and using the algebra (82), one obtains:

$$
S_{\alpha_{2} \gamma 2}^{Z_{1} Z_{2}}\left(p_{1}, p_{2}\right) \prod_{j=3}^{N-1} S_{\alpha_{j} \gamma j}^{\alpha_{j-1} Z_{j}}\left(p_{1}, p_{j}\right) S_{\gamma_{1} \gamma N}^{\alpha_{N-1} Z_{N}}\left(p_{1}, p_{N}\right) \mathrm{e}^{i p_{1} L} \mathcal{F}^{\gamma_{1} \gamma_{2} \ldots \gamma_{N}}=\mathcal{F}^{Z_{1} Z_{2} \ldots Z_{N}}
$$

(summation over repeated indices is implied), that can be shortly rewritten in matrix form as

$$
-\operatorname{Tr}_{0}\left(L_{01}\left(p_{1}, p_{1}\right) L_{02}\left(p_{1}, p_{2}\right) \ldots L_{0 N}\left(p_{1}, p_{N}\right)\right) \mathcal{F}_{N}=\mathrm{e}^{-i p_{k} L} \mathcal{F}_{N}
$$

using the property $S_{\alpha^{\prime} \beta^{\prime}}^{\alpha \beta}(p, p)=-\delta_{\alpha \beta^{\prime}} \delta_{\beta \alpha^{\prime}}$ of the $S$-matrix (100). The matrix $L_{0 k}\left(p_{1}, p_{2}\right)$ is a matrix (100) acting nontrivially in $s u(2)_{0} \otimes s u(2)_{k}$, and acting as identity matrix in the other subspaces from $s u(2)_{0} \prod_{j=1}^{N} \otimes s u(2)_{j}$, and $\operatorname{Tr}_{0}$ denotes trace over the $s u(2)_{0}$. 
Subsequent analysis of the above eigenvalue equations can be done in the framework of standard coordinate [18] or algebraic nested [19] Bethe Ansatz, leading to following spectral equations (we made the transformation (101) to the difference form):

$$
\begin{gathered}
\mathrm{e}^{\eta L}\left(\frac{\operatorname{sh}\left(\lambda_{k}\right)}{\operatorname{sh}\left(\lambda_{k}+\eta\right)}\right)^{L}=(-1)^{N+1} \prod_{n=1}^{N} \frac{\operatorname{sh}\left(\lambda_{k}-\lambda_{n}-\eta\right)}{\operatorname{sh}\left(\lambda_{n}-\lambda_{k}-\eta\right)} \prod_{\epsilon=1}^{N^{B}} \frac{\operatorname{sh}\left(\lambda_{\epsilon}^{(1)}-\lambda_{k}-\eta\right)}{\mathrm{e}^{\eta} \operatorname{sh}\left(\lambda_{\epsilon}^{(1)}-\lambda_{k}\right)} \\
\mathrm{e}^{-\eta N} \prod_{n=1}^{N} \frac{\operatorname{sh}\left(\lambda_{\nu}^{(1)}-\lambda_{n}\right)}{\operatorname{sh}\left(\lambda_{\nu}^{(1)}-\lambda_{n}-\eta\right)}=(-1)^{N_{B}+1} \prod_{\epsilon=1}^{N^{B}} \frac{\operatorname{sh}\left(\lambda_{\epsilon}^{(1)}-\lambda_{\nu}^{(1)}-\eta\right)}{\operatorname{sh}\left(\lambda_{\nu}^{(1)}-\lambda_{\epsilon}^{(1)}-\eta\right)}
\end{gathered}
$$

where $\mathrm{e}^{\eta}=\sqrt{g / h}, k=1,2 \ldots N, \nu=1,2 \ldots N^{B}, N^{B} \leq N$. The above Bethe Ansatz appeared without the proof in 9 .

\section{Case I, some zero hopping rates (tracer diffusion)}

Commuting the term depending on $\left(p_{k}\right)$ the "circle" inside the trace in (110), one has using the (110,104):

$$
\mathcal{F}^{Z_{1}, \ldots Z_{N}}\left(p_{1}, \ldots p_{N}\right)=R_{k}\left(p_{1}, \ldots p_{N}\right) \mathcal{F}^{Z_{N} Z_{1} \ldots Z_{N-1}}\left(p_{1} p_{2} \ldots p_{N}\right)
$$

where

$$
R_{k}\left(p_{1}, \ldots p_{N}\right)=\prod_{j \neq k}^{N} f\left(p_{k}, p_{j}\right) \mathrm{e}^{i p_{k} L}
$$

The set of equations contained in (116) means that all $R_{k}$ are strictly the same, $R_{1}=R_{2}=$ $\ldots R_{N}$. Note additionally that in the right-hand side of Eq.(116), the lower indexes are shifted one step to the the right. It gives the guideline for determining the spectral equation. E.g. if all $Z_{i}=A$, shift of the sequence $\left\{Z_{i}\right\}$ leaves it invariant, so we will obtain $R_{k}=1$, for any $k$. If the sequence $\left\{Z_{i}\right\}$ is periodic with period $\mathrm{n}$, recurrent use of (116) yields $R_{k}^{n}=1$. In the general case, when $\left\{Z_{i}\right\}$ is non-periodic, recurrent use of (116) $N$ times gives:

$$
R_{k}\left(p_{1}, \ldots p_{N}\right)^{N}=1, \quad R_{1}=R_{2}=\ldots R_{N}
$$

\section{Case I, some zero hopping rates (5-vertex model)}

Proceeding analogously to (112), and denoting $a(p)=1-\alpha \mathrm{e}^{i p}, b(p)=1-\beta \mathrm{e}^{i p}$ one obtains:

$$
\begin{gathered}
\left(\alpha \mathrm{e}^{i p_{k}}\right)^{L}=(-1)^{N+1}\left(\frac{\alpha}{\beta}-1\right)^{N_{B}} \prod_{n=1}^{N} \frac{a\left(p_{k}\right)}{a\left(p_{n}\right)} \prod_{\epsilon=1}^{N^{B}} \frac{a\left(p_{\epsilon}^{(1)}\right)-a\left(p_{k}\right)}{a\left(p_{\epsilon}^{(1)}\right) a\left(p_{k}\right)} \\
\left(\frac{\beta}{\alpha}\right)^{L}\left(\frac{\alpha}{\beta}-1\right)^{N} \prod_{n=1}^{N} \frac{a\left(p_{\nu}^{(1)}\right)-a\left(p_{n}\right)}{a\left(p_{\nu}^{(1)}\right) a\left(p_{n}\right)}=(-1)^{N_{B}+1} \prod_{\epsilon=1}^{N^{B}} \frac{a\left(p_{\epsilon}^{(1)}\right)}{a\left(p_{\nu}^{(1)}\right)} \frac{b\left(p_{\nu}^{(1)}\right)}{b\left(p_{\epsilon}^{(1)}\right)}
\end{gathered}
$$

where $k=1,2 \ldots N, \nu=1,2 \ldots N^{B}, N^{B} \leq N$.

Alternatively, one can obtain the nested Bethe Anzatz in this and other cases by an additional (nested) Fourier transform of either $\mathcal{D}_{A}(p)$ or $\mathcal{D}_{B}(p)$, i.e.

$$
\mathcal{D}_{B}(p)=\mathcal{D}_{A}(p) \int \mathrm{e}^{-i q p} \Delta(q) d q
$$


We remark that the Zamolodchikov-type algebra not only leads to the spectral equations (114)-(119) but also implies functional relations for the matrix elements (110). These functional relations are satisfied by Bethe wave functions as in the spin $1 / 2$ case [23] and hence yield expressions for the expectations as integrals over appropriately chosen contour. Alternatively one could search for representations [35] and directly calculate the matrix elements. This procedure requires further investigation.

\section{Acknowledgments}

G.M.S. would like to thank R.B. Stinchcombe and F. Essler for useful discussions and the Department of Physics, University of Oxford, where part of this work was done for providing a stimulating environment. E.F. thanks the Institut für Festkörperforschung for kind hospitality. V.P. acknowledges financial support from the Deutsche Forschungsgemeinschaft. We would like to thank F.C. Alcaraz for interesting discussions.

\section{References}

[1] R.J. Baxter, Exactly solvable models in Statistical Mechanics, Academic Press, London, 1982.

[2] T.M. Liggett, Stochastic Interacting Systems: Contact, Voter ans Exclusion Processes, Springer, Berlin, 1999.

[3] G.M. Schütz, Exactly solvable models for many-body systems far from equilibrium, in: Phase Transitions and Critical Phenomena, Vol. 19, C. Domb and J. Lebowitz (eds.) (Academic Press, London, 2000).

[4] R.B. Stinchcombe and G.M. Schütz, Europhys. Lett. 29, 663 (1995).

[5] R.B. Stinchcombe and G.M. Schütz, Phys Rev.Lett. 75, 140 (1995).

[6] H. Bethe, Z. Phyz. 61, 205 (1931).

[7] P. P. Kulish and E. K. Sklyanin, Lecture Notes in Physics, vol. 151 (Springer, Berlin 1982) p. 61.

[8] F. C. Alcaraz, S. Dasmahapatra and V. Rittenberg, J. Phys. A: Math. Gen. 31845 (1998).

[9] Silvio R. Dahmen, cond-mat/9405031.

[10] P.F.Arndt, T.Heinzel and V.Rittenberg, J. Phys. A: Math. Gen. 31833 (1998).

[11] A. Sudbury and P. Lloyd Ann. Prob.23, 1816 (1995).

[12] T.M. Liggett, Interacting Particle Sistem, Springer, Berlin, 1985.

[13] H. Spohn, Large Scale Dynamics of Interacting Particles Springer, Berlin, 1991.

[14] V. Privman (ed.), Nonequilibrium Statistical Mechanics in One Dimension (Cambridge University Press, Cambridge, 1997). 
[15] B. Schmittmann and R.K.P. Zi Statistical mechanics of Driven Diffusive Systems, in Phase Transitions and Critical Phenomena, vol. 17, ed. C.Domb and J. Lebowitz, Academic Press, New York 1995.

[16] B.Derrida, M.R. Evans, V. Hakim, V. Pasquier J.Phys.A 26, 1493 (1993).

[17] F.C. Alcaraz, private communication

[18] C.N. Yang, Phys. Rev. Lett. 19, 1312-1314 (1967).

[19] P.P Kulish and N.Yu.Reshetikhin, Sov.Phys.JETP 53(1), 108-114 (1981).

[20] V. Popkov and G.M. Schütz, submitted to the Proceedings of the Conference "Theory of Functions and Mathematical Physics", J. Math. Phys., Analysis and Geometry.

[21] Jae Dong Noh and Doochul Kim,Phys. Rev. E 49, 1943-1961 (1994).

[22] V.R. Jones, Int. J. Mod. Phys. B4, 701 (1990).

[23] G.M. Schütz, Eur. Phys. J. B5, 589 (1998).

[24] A. Klumper, A. Schadschneider and J. Zittarz, J. Phys. A 24, L955 (1991)

[25] I. Affleck, T. Kennedy, E.H. Lieb and H. Tasaki, Comm. Math. Phys. 115, 477 (1988).

[26] E.H.Lieb, F.Y.Wu: in "Phase Transitions and Critical Phenomena",Vol.1 (Exact results). Ed. C.Domb, M.Green; Academic Press, N.Y., 1972, p. 436.

[27] A.B. Zamolodchikov, Comm. Math. Phys. 69, 165 (1979), A.B. Zamolodchikov and Al.B. Zamolodchikov, Ann. Phys. 120,(NY) 253 (1979).

[28] T. Sasamoto, M. Wadati, J. Phys. Soc. Japan 66, 279 (1996).

[29] R.B. Stinchcombe, to be published

[30] V. Belitzky and G.M. Schütz, to be published

[31] For another choice of rates this model was studied in [16]

[32] S. Alexander and B. Pincus, Phys. Rev. B 18, 2011 (1978); H. V. Beijeren, K.W. Kehr and R. Kutner, Phys. Rev. B 28, 5711 (1983).

[33] J.E. Santos, Ph.D. Thesis, University of Oxford 1997.

[34] Braid group, knot theory and statistical mechanics C. N. Yang (Ed.), Singapore: World Scientific, 1989.

[35] A. Fring, Int.J.Mod.Phys. A11 1337 (1996); E. Corrigan and P.E. Dorey, Phys. Lett. B273 237 (1991). 